\section{La Adaptación de \\ Materias del Ámbito \\ Científico al Crédito \\ Europeo en las \\ Universidades de la \\ Comunidad Autónoma \\ de Galicia}

UT. Revista de Ciències de l'Educació

Juny 2010. Pag. 55-74

ISSN 0211-3368

http://pedagogia.fcep.urv.cat/revistaut

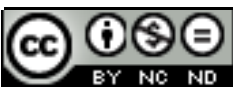

\title{
$M^{\mathrm{a}}$ Elena Añel Cabanelas ${ }^{\mathrm{a}}$
}

Rebut: 07/05/2010 Acceptat: 10/06/2010

\section{Resumen}

El presente artículo pretende hacer una descripción, a modo de reseña bibliográfica, de aquellas publicaciones editadas por la Agencia para la Calidad del Sistema Universitario de Galicia (ACSUG) que surgieron luego de la puesta en práctica de una serie de experiencias piloto para la adaptación de materias al Espacio Europeo de Educación Superior y por lo tanto de adaptación a los nuevos sistemas de créditos ECTS (European Credit Transfer System).

En este caso los documentos reseñados se refieren a titulaciones que se imparten en las tres universidades gallegas (Universidad de Coruña, Universidad de Santiago de Compostela y Universidad de Vigo). Todas ellas son titulaciones del ámbito científico: Ingeniería Técnica de Obras Públicas, Matemáticas, Biología e Informática.

Palabras clave: ECTS, proyecto ACSUG, titulaciones científicas

\section{The Adjustment of Matters of the Scientific Area to the European Credit}

\section{Abstract}

The present article tries to do a description, like bibliographical review, those publications edited by the Agency for the Quality of the University System of Galicia (ACSUG) that arose after the putting in practice of a series of pilot experiences for the

\footnotetext{
a Universidad de Vigo
} 
adjustment of matters to the European Space of Top Education and therefore of adjustment to the new systems of credits ECTS (European Credit Transfer System).

In this case the outlined documents say to qualifications that they are given in three Galician universities (University of Corunna, University of Santiago de Compostela and University of Vigo). All of them are qualifications of the scientific area: Technical Engineering of Public, Mathematical Works, Biology and Computer science.

Keywords: ECTS, project ACSUG, scientific qualifications

\section{1.- El espacio europeo de educación superior (EEES)}

La enseñanza superior se sitúa en el cruce de la investigación, la educación y la innovación y constituye un eje central de la economía y de la sociedad del conocimiento y un factor clave para la competitividad de la Unión Europea.

La sociedad española necesita que su sistema universitario se encuentre en las mejores condiciones posibles de cara a su integración en el Espacio Europeo de Educación Superior.

Se establecen nuevos mecanismos para el fomento de la excelencia como son: mejorar la calidad de la docencia y la investigación, a través de un nuevo sistema objetivo y transparente que garantice el mérito y la capacidad en la selección y el acceso del profesorado y mejorar, asimismo, la calidad de la gestión, mediante procedimientos que permitirán resolver con agilidad y eficacia las cuestiones de coordinación y administración de la Universidad.

El Espacio Europeo de Educación Superior es un ámbito de organización educativo iniciado en 1999 con la Declaración de Bolonia que quiere integrar los distintos sistemas educativos de la Unión Europea y proporcionar una forma eficaz de intercambio entre todos los estudiantes así como dotar de una dimensión y de una agilidad sin precedentes al proceso de cambio emprendido por las universidades europeas. Para ello, se basa en los siguientes aspectos fundamentales: los créditos ECTS (European Credit Transfer System), la estructura grado, postgrado/máster, la acreditación y el suplemento al título.

El denominado EEES se apunta como el marco de organización educativa más importante de las próximas décadas, el año 2010 es el año previsto por la citada Declaración para la plena consecución de sus objetivos.

Pensando en el futuro actualmente España está inmersa en la denominada "Estrategia Universidad 2015" "EU2015" (http://www.educacion.es/eu2015). Se trata de una iniciativa del Gobierno de España encaminada a la modernización de las universidades españolas mediante la coordinación de los correspondientes sistemas universitarios autonómicos y el desarrollo de un moderno Sistema Universitario Español. Esta iniciativa, impulsada 
inicialmente en 2008 por el Ministerio de Ciencia e Innovación, está hoy pilotada por el Ministerio de Educación a través de la Secretaría General de Universidades, con el apoyo de las correspondientes consejerías de las Comunidades Autónomas, así como de las propias universidades.

La EU2015 determina la responsabilidad académica colectiva para promover las mejoras necesarias que faciliten su eficiencia y eficacia sociales e incremente su contribución socioeconómica, manteniendo los principios básicos de autonomía universitaria, libertad académica, rendición de cuentas a la sociedad y espíritu crítico.

Esta iniciativa persigue que las universidades estén académicamente más preparadas, sean más eficientes y estén más internacionalizadas para afrontar el futuro; unas universidades que presten mayor atención a la colaboración interuniversitaria, a la agregación estratégica con otras instituciones y agentes; unas universidades más reconocidas socialmente y más valoradas y mejor financiadas por las correspondientes administraciones y por la sociedad.

Los ejes iniciales de la EU2015 se agruparon en cuatro ámbitos: misiones, personas, capacidades y entorno, dentro de los que se han diseñado líneas estratégicas que en conjunto abarcan los principales retos de la Agenda Europea de Modernización de las Universidades, así como dan respuesta a los problemas más específicos del Sistema Universitario Español.

\section{2.- Los créditos ECTS (European Credit Transfer System)}

En el Real Decreto 1125/2003 de 5 de septiembre (B.O.E. de 18 de septiembre de 2003) se establece el sistema europeo de créditos y el sistema de calificaciones en las titulaciones universitarias españolas. En este marco normativo se define el crédito ECTS como una unidad de valoración de la actividad académica en la que se integran enseñanzas teóricas y prácticas, así como otras actividades académicas dirigidas y el volumen de trabajo que el estudiante debe realizar para alcanzar los objetivos educativos.

Por lo tanto esta nueva unidad de medida representa la cantidad de trabajo del estudiante para cumplir los objetivos del programa. Incluye las horas de clase, las horas de estudio, la elaboración de trabajos o la realización de pruebas entre otros, por lo que el examen tradicional pierde importancia frente a la evaluación continua. Aproximadamente un crédito ECTS sería equivalente a 25-30 horas de aprendizaje del estudiante, lo que supone una marcada diferencia con la unidad de crédito tradicional que hasta ahora se venía utilizando en el sistema educativo universitario.

Muñoz Camacho y otros (2004:17) dicen lo siguiente: "La decisión de adoptar un sistema europeo basado en créditos viene dado por la necesidad de dar respuesta a tres exigencias básicas:

- La transparencia y la comparabilbidad de la información sobre los programas de estudios y los resultados de los estudiantes. 
- El acuerdo mutuo entre los centros asociados y los estudiantes para el reconocimiento de los estudios realizados en el extranjero.

- La definición de un volumen de trabajo efectivo del estudiante para la consecución de los objetivos de aprendizaje y de las competencias del programa de estudios.

Las publicaciones que se reseñan en el presente artículo están directamente relacionadas con la aplicación de los créditos ECTS en el Sistema Universitario de Galicia.

\section{3.- Las agencias de calidad}

Una de las principales innovaciones de la Ley Orgánica de Universidades (B.O.E. 24 de diciembre de 2001), conocida como LOU, viene dada por la introducción en el sistema universitario de mecanismos externos de evaluación de su calidad creando la Agencia Nacional de Evaluación de la Calidad y Acreditación, conocida como ANECA. De manera independiente, esta agencia desarrolla la actividad evaluadora propia de sistemas universitarios avanzados y necesaria para medir el rendimiento del servicio público de la enseñanza superior y reforzar su calidad, transparencia, cooperación y competitividad. La agencia evalua tanto las enseñanzas como la actividad investigadora, docente y de gestión, así como los servicios y programas de las universidades. Su trabajo proporcionará una información adecuada para la toma de decisiones, tanto a los estudiantes a la hora de elegir titulaciones o centros como a los profesores y a las administraciones públicas al elaborar las políticas educativas que les corresponden.

La LOU posibilitó también la creación de órganos de evaluación de la enseñanza superior en las distintas Comunidades Autónomas, fruto de ello surgen en distintos momentos otras agencias que a nivel autonómico establecen también mecanismos de actuación en este campo.

En la Comunidad Autónoma de Galicia empieza a trabajar la Agencia para la Calidad del Sistema Universitario de Galicia (ACSUG). Se crea el 30 de enero de 2001 bajo la figura jurídica de un consorcio entre el Xunta de Galicia y las tres universidades gallegas, nace con la voluntad de conseguir que el Sistema Universitario de la Comunidad alcance un gran prestigio y reconocimiento dentro del contexto universitario nacional, europeo e internacional.

La integración de las tres universidades gallegas (Universidad de Coruña, Universidad de Santiago de Compostela y Universidad de Vigo) en el Espacio Europeo de Educación Superior supone un reto de enorme trascendencia para el futuro de nuestra sociedad. Desde la ACSUG (http://www.acsug.es) se impulsa un Proyecto Piloto de implantación del crédito ECTS en distintas titulaciones con el objetivo de que toda la comunidad académica no solo se familiarizara, sino que integrara la nueva terminología y los principios básicos del EEES. Su pretensión era extraer 
conclusiones, orientar y aportar ideas al debate surgido sobre el establecimiento del crédito europeo y del proceso de adecuación de las enseñanzas a este nuevo modelo.

En el curso académico 2003/2004 se promueven desde la Agencia varias experiencias piloto de experimentación del crédito europeo dentro de algunas titulaciones del Sistema Universitario de Galicia, derivadas de la implementación de estas experiencias surgen una serie de publicaciones tanto del ámbito jurídico-social como del ámbito científico objeto de análisis en el presente artículo.

Cabe apuntar que estas publicaciones responden a una estructura similar en la que se alude al diseño del proyecto, su desarrollo y su evaluación en las distintas titulaciones. Decir por otro lado que todas ellas están editadas únicamente en lengua gallega aunque la reseña que aquí se presenta sea en lengua castellana.

El esquema seguido para la descripción de las publicaciones consta de cuatro puntos: la referencia bibliográfica, la ficha técnica, el centro, titulación y universidad a la que pertenece y por último la descripción del contenido.

\section{4.- Publicación referida a la titulación de ingeniero técnico de obras públicas}

Ingeniería Técnica de Obras Públicas fue una de las titulaciones que participó en este proyecto, cuya experiencia se conoce a través de la monografía publicada posteriormente. La publicación responde a la siguiente referencia:

AAVV (2007). Adaptación de materias al crédito europeo. Titulación de Ingeniero Técnico de Obras Públicas. Proyecto ACSUG, Santiago de Compostela: ACSUG

La ficha técnica que recoge los datos de la publicación sería la siguiente:

Título: Adaptación de materias al crédito europeo. Titulación de Ingeniero

Técnico de Obras Públicas. Proyecto ACSUG

Páginas: 31

Año: 2007

Depósito legal: C-2171-2007

Figura 1: Ficha técnica - Ingeniero Técnico de Obras Públicas 


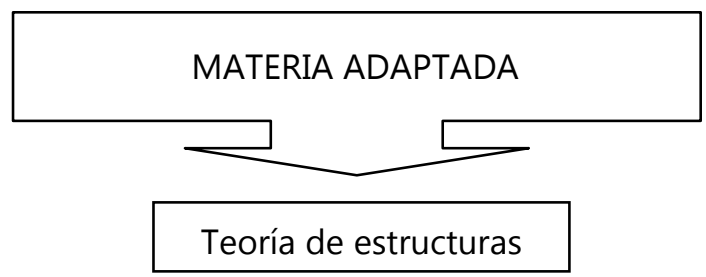

La experiencia de implantación del EEES se llevó a cabo en la materia de Teoría de Estructuras que pertenece a:

Titulación: Ingeniero de obras públicas. Especialidad en construcciones civiles.

Centro: Escuela Técnica Superior de Ingenieros de caminos, canales y puertos.

Universidad: La Coruña

El contenido de la publicación que describe la experiencia de implantación de los créditos ECTS en esta titulación se estructura en siete grandes apartados:

- Una breve Introducción en la que se explica el origen de su incorporación al proyecto de la ACSUG, éste fue por iniciativa de dos profesores que impartían la materia "Teoría de estructuras" y que apostaban por llevar a cabo actividades de innovación educativa decidiendo así trabajar en la adaptación de la materia al EEES. Esta materia se impartía por primera vez en el curso 2004-2005 y sería cursada por todo el alumnado ya que era troncal.

- Un apartado denominado Diseño del proyecto de adaptación en el que facilitan datos concretos sobre la materia que se adaptará: número de créditos (6 teóricos y 4,5 prácticos), curso en el que se imparte $\left(2^{\circ}\right)$ o la especialidad a la que pertenece entre otros (construcciones civiles).

- $\quad$ El tercer punto se titula Desarrollo de la experiencia [1] en él se identifica al profesorado involucrado en la experiencia piloto, se alude a la documentación tomada como base para la elaboración de la guía docente y por último se describe la metodología que se empleará en la materia justificando la necesidad de la clase magistral y la utilización de sitios web elaborados por los profesores, también se describe el sistema de evaluación empleado.

- El siguiente apartado se denomina Desarrollo de la experiencia [2] y consta de siete subapartados:

- La descripción de la situación de la materia en el plan de estudios, objetivos y carga docente así como la evolución previa de la materia: se 
alude al nombre, código o tipo de materia, el ciclo y curso, su número de créditos o los objetivos que se plantean en la misma. En cuanto a su evolución previa no se facilitan datos porque la adaptación se produce en el primer curso académico en el que se imparte.

- Diseño de la adaptación. Nuevas metodologías docentes (objetivos y diseño): se plantean poner en práctica una metodología basada en las clases magistrales, valorando el esfuerzo y la capacidad de trabajo del alumnado a través de una web a disposición de los estudiantes así como la presentación de trabajos o las actitudes que presentan.

- Cuantificación del trabajo del alumno: se lleva a cabo esta cuantificación a través de encuestas realizadas en el aula con el objetivo de determinar la cantidad de horas dedicadas a la materia y su distribución.

- Métodos de evaluación: de forma general se pretende evaluar el grado de interiorización de los contenidos de la materia por parte del alumnado, para ello se tocan tres aspectos: los conceptos evaluados a través de un examen, la asistencia a clase y participación cuantificadas a través de observaciones y notas del profesorado o los trabajos personales presentados que tendrán una valoración específica.

- Resultados académicos: aquí se recogen los resultados alcanzados por los estudiantes en los cursos 2004-2005 y 2005-2006 diferenciando el número de matriculados, el de presentados a cualquier convocatoria y el de aprobados en cualquier convocatoria.

- Encuestas de esfuerzo y de opinión de alumnado: en los dos cursos académicos referidos en el punto anterior se llevan a cabo encuestas en las que se detecta que un gran número de alumnos no dedican ningún tiempo de trabajo a la materia, exceptuando la asistencia a clase, por lo que se hace difícil extraer conclusiones. De todas formas con los datos que proporcionan dichas encuestas se menciona que en término medio el alumnado que sigue la materia le dedica un tiempo de trabajo personal de unas 200 horas y que el grado de satisfacción con la adaptación de la materia se considera alto.

- Evaluación de la experiencia por el profesorado y perspectivas del futuro: en general la evaluación de esta experiencia piloto es positiva, aluden a las ventajas de la utilización de sitios web, la necesidad de seguir esforzándose para mejorar aspectos concretos y la importancia de las tutorías como un aspecto fundamental para personalizar el proceso de enseñanza-aprendizaje de los estudiantes.

- El quinto punto se dedica a la Evaluación global del proceso de experimentación y en el se recogen distintos aspectos relacionados con: 
- Aspectos de la organización de los centros: el profesorado implicado en la puesta en marcha de esta experiencia hizo lo posible para minimizar el impacto en el funcionamiento cotidiano del centro, objetivo conseguido ya que no se mencionan interferencias o incidencias sino la existencia de una colaboración fructífera y armoniosa en todo momento.

- Cuestiones docentes y académicas: desde el principio de la propuesta de adaptación el profesorado intentó que los cambios fueran de poca magnitud, aprovechando los aspectos más innovadores o de mejora de calidad que ofrece el ECTS, con esta premisa se tomaron las precauciones necesarias para no interferir o distorsionar el desarrollo habitual de la materia, entre las medidas adoptadas se mencionan la creación de un sistema personalizado de tutorías para profundizar en las necesidades de cada estudiante o la realización de trabajos personales al final de cada tema.

- Síntesis de las opiniones del alumnado: a través de encuestas anónimas hechas con periodicidad variable se deduce una posición favorable aunque cautelosa del alumnado.

- Valoraciones sobre la experimentación: la valoración de la experiencia es positiva ya que permitió ensayar en condiciones realistas la adaptación de la materia al EEES. Por otro lado en este punto los autores destacan la necesidad de que los cambios que se lleven a cabo en las titulaciones de tipo técnico han de estar solidamente fundamentadas y contrastadas y que además deben garantizar mejoras seguras en el proceso de aprendizaje de los estudiantes sin poner en riesgo la gran eficacia que a lo largo de los años se produce en nuestro país en cuanto a la preparación de profesionales en este campo.

- El penúltimo apartado se denomina Recomendaciones sobre necesidades de organización, de recursos y de cambios de gestión y en él se expone a necesidad de llevar a cabo campañas informativas para el alumnado en cuanto a las novedades de la adaptación de las materias, la necesidad de una mayor dotación de recursos tanto materiales como humanos en los centros y la atención y respuesta a las consecuencias de un aumento considerable del trabajo docente.

- Para finalizar la publicación se incluyen dos Apéndices en el primero se muestran las presentaciones empleadas en el proceso de implantación y de difusión de la experiencia a través de siete diapositivas y un segundo apéndice formado por la guía docente de la materia en proceso de adaptación.

\section{5.- Publicación referida a la titulación de matemáticas}

Matemáticas fue también una de las titulaciones que participó en este proyecto, en la publicación objeto de análisis además del punto de vista del alumnado, profesorado y órganos de gobierno implicados se contemplan los resultados obtenidos y las 
trabas encontradas en la elaboración y sobre todo en la puesta en marcha o desarrollo de las nuevas guías docentes. La referencia de dicha publicación es la siguiente:

AAVV (2007). Adaptación de materias al crédito europeo. Titulación de Matemáticas. Proyecto ACSUG, Santiago de Compostela: ACSUG.

La ficha técnica que recoge los datos de la publicación sería la siguiente:

Título: Adaptación de materias al crédito europeo. Titulación de Matemáticas.

Proyecto ACSUG

Páginas: 296

Año: 2007

Depósito legal: C-2656-2007

ISBN: 978-84-611-8197-1

Figura 2: Ficha técnica - Matemáticas

\section{MATERIAS ADAPTADAS}

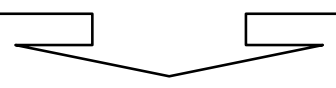

\begin{tabular}{|l|l|}
\hline Curso & Materia \\
\hline $1^{\circ}$ & Álgebra linear y multilinear \\
\hline $1^{\circ}$ & Cálculo diferencial e integral \\
\hline $1^{\circ}$ & Informática \\
\hline $1^{\circ}$ & Introducción al cálculo numérico \\
\hline $1^{\circ}$ & Topología de los espacios euclidianos \\
\hline $1^{\circ}$ & Introducción al análisis matemático \\
\hline $1^{\circ}$ & Geometría métrica \\
\hline $2^{\circ}$ & Análisis numérica matricial \\
\hline $2^{\circ}$ & Diferenciación de funciones de varias variables reales \\
\hline $2^{\circ}$ & Integración de funciones de V.V. reales \\
\hline $2^{\circ}$ & Introducción a las ecuaciones diferenciales ordinarias \\
\hline $2^{\circ}$ & Introducción al cálculo de probabilidades \\
\hline $2^{\circ}$ & Geometría afín y proyectiva \\
\hline $2^{\circ}$ & Topología \\
\hline $3^{\circ}$ & Curvas y superficies \\
\hline $3^{\circ}$ & Elementos de variable compleja \\
\hline $3^{\circ}$ & Inferencia estadística \\
\hline $3^{\circ}$ & Introducción al álgebra \\
\hline $3^{\circ}$ & Métodos numéricos \\
\hline $3^{\circ}$ & Series de Fourier e introducción a las E.D.P. \\
\hline
\end{tabular}




\begin{tabular}{|l|l|}
\hline $3^{\circ}$ & Teoría global de superficies \\
\hline $3^{\circ}$ & Vectores aleatorios \\
\hline $4^{\circ}$ & Cálculo numérico \\
\hline
\end{tabular}

La experiencia de implantación del EEES se llevó a cabo en 23 materias de las que 16 son troncales y obligatorias del primer ciclo (de un total de 22) y el resto son optativas o troncales del segundo ciclo. Se involucra profesorado de varios departamentos de la titulación de matemáticas de un centro concreto:

Titulación: Licenciado en matemáticas

Centro: Facultad de matemáticas.

Universidad: Santiago de Compostela

El contenido de la publicación que describe la experiencia de implantación de los créditos ECTS en esta titulación se estructura en seis grandes apartados:

- El primero de ellos dedicado a la descripción de La Facultad de Matemáticas de la Universidad de Santiago de Compostela en el que podemos encontrar un recorrido histórico sobre la titulación impartida en esta facultad desde mediados del siglo XVIII a la actualidad incluyendo aspectos como: los grupos de investigación existentes, publicaciones, iniciativas llevadas a cabo con la finalidad de aumentar el número de matriculados o medidas de renovación de la titulación entre las que se destaca la participación del profesorado en el Programa de experiencias piloto de adaptación al EEES promovido por la ACSUG. En lo que se refiere a esta medida concreta destacar que la publicación incluye los resultados de una encuesta sobre el trabajo del alumnado realizada en la Facultad durante los cursos 2000-2001 y 2001-2002, es decir, en cursos anteriores a la realización de las experiencias (cursos 2004-2005 y 2005-2006), así como los resultados de una encuesta para el profesorado con el objetivo de contrastar el trabajo de los estudiantes con lo que el profesorado cree que es necesario que realicen.

- Un segundo apartado titulado Diseño y desarrollo general del proyecto de experiencias piloto de adaptación al EEES habla del proceso de incorporación de la Facultad a este proyecto marcado por la participación voluntaria del profesorado así como las líneas de actuación en el curso 2004-2005 y en el 2005-2006. Entre las primeras se diferencian por un lado actividades de información y formación del profesorado entre las que podemos encontrar cursos, conferencias, sesiones de trabajo o la realización y publicación de una memoria sobre la situación de la Facultad de Matemáticas y por otro lado actividades del profesorado que participa en la experiencia de 
adaptación de materias como la redacción de un proyecto de experiencia, de una guía docente, controlar el tiempo de trabajo invertido por el alumnado en su materia, potenciar la asistencia a seminarios y tutorías, no limitar la evaluación a los exámenes finales, asistir a las actividades y reuniones programadas para el seguimiento de la experiencia piloto y por último redactar una memoria final con los resultados o conclusiones obtenidos. En cuanto a las líneas de actuación para el curso 2005-2006 se dividen de nuevo en actividades de apoyo, información y formación así como las expuestas anteriormente relacionadas con el profesorado involucrado en la adaptación de materias.

- El tercero Desarrollo del proyecto: guías docentes, proyectos y memorias de las experiencias materia a materia un apartado que está totalmente desarrollado en el Anexo II del documento al que se nos remite.

- A continuación un cuarto apartado recoge la Evaluación global del proceso de experiencia piloto en él se alude por un lado al Anexo I en el que se recogen algunos datos interesantes para la evaluación y por otro lado se describen aquellas conclusiones, valoraciones y comentarios que se desprenden de los informes individuales enviados por el profesorado de cada materia y las propias consideraciones del equipo decanal que se resumen en la existencia de una satisfacción generalizada y una valoración positiva sobre las experiencias de adaptación al EEES.

- Un Anexo I en el que se puede ver el informe de rendimiento académico referente al primer ciclo de los cursos 2003-2004, 2004-2005 y 2005-2006, una parte importante de la evaluación global y del informe de la experiencia en el que hace un tratamiento semejante para cada materia y cada año académico haciendo una comparación absoluta y porcentual de los siguientes ítems:

- Número de alumnos que superaron la materia en la primera convocatoria frente al número de alumnos matriculados.

- Número de alumnos que superaron la materia en segunda convocatoria frente a los que estaban pendientes de aprobarla.

- Número de alumnos que superaron la materia (en cualquier convocatoria) frente al número total de matriculados.

- Un último Anexo /l que alude a la descripción materia a materia de las experiencias piloto de adaptación al EEES en los cursos 2004-2005 y 20052006 siguiendo un esquema sugerido por la ACSUG incorporando de cada materia los siguientes documentos elaborados por el profesorado responsable: 
- Guía docente de la materia, que figura en la programación general del centro. En muchos casos esta guía docente coincide con el proyecto de la experiencia piloto de la materia.

- Proyecto de experiencia aprobado en su día por la Comisión de Docencia del Centro, que se omite en los casos que coincide con la guía docente.

- Informe o memoria de conclusiones de la experiencia también con el visto bueno de la Comisión de Docencia.

\section{6.- Publicación referida a la titulación de biología}

Biología fue otra de las titulaciones de ámbito científico que participó en el proyecto de adaptación de materias al crédito europeo promovido desde la ACSUG. El proceso de implementación de dicho proyecto se recoge en la presente publicación que responde a la siguiente referencia bibliográfica:

AAVV (2007). Adaptación de materias al crédito europeo. Titulación de Biología. Proyecto ACSUG, Santiago de Compostela: ACSUG.

La ficha técnica que recoge los datos de la publicación sería la siguiente:

Título: Adaptación de materias al crédito europeo. Titulación de Biología.

Proyecto ACSUG

Páginas: 102

Año: 2007

Depósito legal: C-2171-2007

Figura 3: Ficha técnica - Biología

\begin{tabular}{|l|}
\hline Citología e Histología Vegetal y Animal \\
\hline Etología \\
\hline Neurobiología general y comparada \\
\hline Citología \\
\hline Histología Vegetal y Animal \\
\hline Comportamiento animal \\
\hline Microbiología y Biotecnología Ambiental \\
\hline
\end{tabular}




\begin{tabular}{|l|}
\hline Genética Molecular \\
\hline Etología \\
\hline Fitogeografía \\
\hline Bioquímica I \\
\hline Métodos de ecología \\
\hline Conservación y explotación de recursos animales \\
\hline Biología celular \\
\hline Ingeniería de procesos fermentativos \\
\hline Microbiología industrial \\
\hline Biotecnología vegetal \\
\hline
\end{tabular}

La experiencia de implantación del EEES se llevó a cabo en:

Titulación: Licenciatura en Biología

Centro: Facultades de Biología y Facultad de Ciencias

Universidades: Santiago de Compostela, Vigo y La Coruña.

La publicación que describe la experiencia de implantación de los créditos ECTS en esta titulación se estructura en los siguientes apartados:

- Presentación en la que el director de la ACSUG describe los objetivos del proyecto promovido desde la agencia que dirige y la incorporación de las facultades mencionadas a dicho proyecto.

- Prólogo en el que el decano de la Facultad de Biología de la Universidad de Santiago como coordinador de la experiencia alude a la importancia de la integración de las universidades gallegas al EEES, la invitación recibida por parte de la ACSUG y la incorporación al proyecto.

- El Origen del proyecto, en este apartado se incluyen aspectos generales relacionados con la construcción del EEES o cuál es el origen de proyecto impulsado por la ACSUG y por otro lado haciendo referencia específica a la titulación de biología se hace referencia a la relación de docentes implicados en la adaptación de materias al crédito europeo, cuáles son las experiencias propias de cada universidad o cuál es el mapa de la titulación. Para acabar el apartado se citan otras experiencias llevadas a cabo en distintas titulaciones de las tres universidades gallegas.

- El Diseño del proyecto de adaptación de los estudios de Biología al EEES. Este apartado describe cuál fue el proceso de implicación de las distintas Facultades de Biología en el proyecto de adaptación al EEES y cómo se desarrolló el primer proyecto y su resultado en el que se distinguen dos fases, la primera de ellas la implantación de materias piloto y la segunda la 
elaboración de una propuesta de plan de estudios conducente a la obtención del título de grado. Por otro lado en este apartado también se contempla cómo se acordó la elección de las materias y la posterior configuración de grupos de trabajo, cuál fue el sistema de coordinación interuniversitaria y las actividades que se realizaron (reuniones, intercambio de ideas, jornadas de debate,...) y por último se describe brevemente cuál es la situación actual y las perspectivas de futuro.

- Las Actividades de formación; se llevaron a cabo éstas con el objetivo de dar a conocer al profesorado implicado la terminología, los principios básicos del EEES o las experiencias de otras universidades. En esta publicación se hace una recopilación del listado de actividades implementadas que se pueden agrupar en conferencias y seminarios, talleres de trabajo y reuniones.

- Un cuarto punto denominado Desarrollo de la experiencia explica que en el proyecto piloto participaron un total de 26 docentes de 17 materias (que se repiten en varios cursos) y que el alumnado varió entre 125 y 240 participantes en las materias de primer ciclo y entre 16 y 53 en las de segundo ciclo. En todos los casos se utilizó un modelo base de guía docente con los siguientes puntos:
a. Nombre y código de la materia
b. Curso en el que se imparte según el plan de estudios
c. Tipo de materia (troncal, obligatoria, optativa)
d. Número de créditos ECTS
e. Lengua en la que se imparte
f. Profesores y coordinador
g. Objetivos de la materia
h. Conocimientos previos necesarios y/o recomendados
i. Metodología de aprendizaje
j. Programa de la materia especificando los contenidos teóricos y prácticos
k. Bibliografía básica y complementaria
I. Sistema y criterios de evaluación
m. Horario de clases teóricas y prácticas
n. Horario de seminarios
o. Horario de tutorías especializadas o generales 
Lo que resta del apartado cuatro contiene una descripción detallada del desarrollo de la experiencia en cada una de las materias y los resultados obtenidos tanto académicos como en las encuestas realizadas al alumnado. Cada subapartado lo forma una materia y está elaborado por el profesorado implicado en ella, podemos encontrar las siguientes:

- Citología e Histología Vegetal y Animal

- Evaluación del desarrollo de la experiencia de adaptación al EEES (Material de citología)

- Etología

- Comportamiento animal (Optativa de $4^{\circ}$ y $5^{\circ}$ curso)

- Neurobiología general comparada

- Microbiología y Biotecnología Ambiental

- Genética Molecular

- Evaluación y perspectivas; en este punto se aborda una evaluación global de la experiencia así como una serie de recomendaciones sobre la necesidad de recursos y cambios de gestión. En primer lugar se aportan algunas ideas sobre un modelo de universidad adaptado al EEES que pasaría por construir una universidad de calidad que proporcione una buena formación intelectual y facilite la inserción profesional, la libertad y la responsabilidad de los estudiantes. A continuación se exponen las oportunidades y amenazas del EEES, se entiende que éste debería ser una oportunidad de mejora de la calidad de la docencia universitaria y se critica un proceso de adaptación demasiado mecánico con falta de reflexión. Por otro lado se recoge una propuesta de estrategia y un plan de trabajo para la implementación de la adopción de materias formado por actuaciones como: la creación de equipos de asesores docentes, la elaboración de una primera evaluación en cada centro y de su potencial de adaptación, actividades intensivas de formación sobre EEES e innovación docente, elaboración de un plan estratégico de adaptación, discusión y modificación de dicho plan, organización de grupos de trabajo dentro de las titulaciones para diseñar y coordinar la experiencia y por último el trabajo independiente de los grupos del centro. Se aportan por otro lado algunas ideas sobre el problema de la participación activa de los estudiantes, la necesidad de recursos humanos y materiales o la convicción de que la coordinación es una de las claves del éxito en el proceso de adaptación.

- Conclusiones; la valoración de la experiencia se recoge como muy positiva y se hace un análisis desde la perspectiva de los tres actores principales: el profesorado, el alumnado y los órganos de gobierno de las universidades. 
- Para finalizar se incluye un Anexo en el que se expone el listado de conferencias, talleres y congresos sobre EEES en los que se presentó la experiencia aquí detallada.

\section{7.- Publicación referida a la titulación de informática}

La titulación de Informática se involucró también en el proyecto de la ACSUG. A través de este documento hace público el informe que describe la experiencia de la adaptación de determinadas materias a los nuevos créditos europeos. La referencia de esta monografía es:

AAVV (2007). Adaptación de materias al crédito europeo. Titulación de Informática. Proyecto ACSUG., Santiago de Compostela: ACSUG

La ficha técnica que recoge los datos de la publicación sería la siguiente:

Título: Adaptación de materias al crédito europeo. Titulación de Informática. Proyecto ACSUG

Páginas: 127

Año: 2007

Depósito legal: C-2654-2007

ISBN: 978-84-611-8203-9

Figura 4: Ficha técnica - Informática

\section{MATERIAS ADAPTADAS}

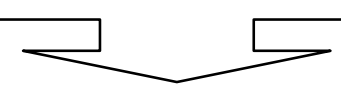

\begin{tabular}{|l|l|}
\hline CURSO & MATERIA \\
\hline $1^{\circ}$ & Álgebra \\
\hline $1^{\circ}$ & Estructura de datos y de la información \\
\hline $1^{\circ}$ & Tecnología de ordenadores \\
\hline $1^{\circ}$ & Bases de datos I \\
\hline $2^{\circ}$ & Algoritmos \\
\hline $2^{\circ}$ & Ingeniería del software de gestión \\
\hline $2^{\circ}$ & Sistemas conexionistas \\
\hline $2^{\circ}$ & Tecnología de la programación \\
\hline $3^{\circ}$ & Medios de transmisión \\
\hline $3^{\circ}$ & Teoría de autómatas y lenguajes formales \\
\hline optativa & Informática biomédica \\
\hline
\end{tabular}




\begin{tabular}{|l|l|}
\hline optativa & Medios de transmisión \\
\hline optativa & Técnicas de simulación \\
\hline
\end{tabular}

La experiencia de implantación del EEES se llevó a cabo en trece materias de:

Titulación: Ingeniería Técnica de Sistemas e Ingeniería Técnica de Gestión

Centro: Facultad de Informática.

Universidad: La Coruña

El contenido de la publicación que describe la experiencia de implantación de los créditos ECTS en esta titulación se estructura en cuatro grandes apartados presentados luego del Prólogo y la identificación del Personal participante:

- $\quad$ El primero de ellos se refiere a los Estudios de Informática en el que se hace un recorrido por las características de éstos a nivel estatal y autonómico haciendo una mención específica de la Facultad de Informática de la Universidad de La Coruña y se describe de forma muy general cómo esta llega a formar parte de las experiencias piloto de adaptación al EEES promovidas desde la ACSUG.

- En segundo lugar se presenta el Desarrollo de la experiencia. Parte I en el que se hace una introducción de los aspectos generales de la experiencia como son el personal implicado, la selección de materias, el programa piloto o el modelo de guías docentes. Se presenta también el modelo utilizado para estimar la carga docente asociada a la implantación de las guías.

- El tercer apartado se denomina Desarrollo de la experiencia. Parte II, en este capítulo se hace una descripción de la experiencia de adaptación realizada en cada materia concretando de cada una de ellas los datos generales que la identifican, el grado de implantación, las actividades desarrolladas, los criterios de evaluación y los resultados obtenidos. Se finaliza cada sección con una estimación de la carga docente y una valoración de la experiencia llevada a cabo entendiendo por sección la parte dedicada a cada materia.

- Un último apartado incluye las Valoraciones generales de la implementación de la experiencia. Desde la facultad se reconoce que no fue una tarea fácil pero que la valoración general de la participación en el proyecto piloto es positiva ya que permitió detectar las fortalezas y debilidades del centro de cara a la implantación de los créditos ECTS. Como conclusión final exponen la intención de ampliar la adaptación de materias a un curso completo y que se impartan dos programas de posgrado en el siguiente curso académico concebidos ambos dentro del EEES. 


\section{8.- Conclusiones}

Las cuatro guías descritas a lo largo del artículo responden como hemos visto a una estructura muy similar. El objetivo de su publicación es divulgar la experiencia llevada a cabo en alguna de las titulaciones de las tres universidades gallegas exponiendo los pasos previos que cada facultad ha realizado, cómo se implementa la adaptación de materias una vez realizado el diseño y por último cuáles fueron los resultados obtenidos después de una valoración objetiva a través de distintos instrumentos de evaluación.

Con estas publicaciones se completa parte del proyecto piloto de implantación de los créditos ECTS en distintas titulaciones haciendo que toda la comunidad académica no solo se familiarice sino que integre la nueva terminología y los principios básicos del Espacio Europeo de Educación Superior.

\section{Referencias}

AAVV (2007). Adaptación de materias al crédito europeo. Titulación de Ingeniero Técnico de Obras Públicas. Proyecto ACSUG, Santiago de Compostela: ACSUG

AAVV (2007). Adaptación de materias al crédito europeo. Titulación de Matemáticas. Proyecto ACSUG, Santiago de Compostela: ACSUG.

AAVV (2007). Adaptación de materias al crédito europeo. Titulación de Biología. Proyecto ACSUG, Santiago de Compostela: ACSUG.

AAVV (2007). Adaptación de materias al crédito europeo. Titulación de Informática. Proyecto

De Miguel, M. (coord.) (2006). Metodologías de enseñanza y aprendizaje para el desarrollo de competencias: orientaciones para el profesorado universitario ante el espacio europeo de educación superior. Madrid : Alianza

González Sanmamed, M (dir) (2006). O EEES: perspectiva do alumnado das Universidades Galegas, Santiago de Compostela: ACSUG.

González Sanmamed, M (dir) (2006). O EEES: perspectiva do profesorado das Universidades Galega. Santiago de Compostela: ACSUG.

Muñoz Camacho, E. y otros (2004). O Espazo Europeo de Educación Superior: aspectos xerais, Santiago de Compostela: ACSUG.

Ministerio de Educación del Gobierno de España (25/05/2010). ¿Qué es la Estrategia Universidad 2015? Recuperado de http://www.educacion.es/eu2015

Ministerio de Educación (2009) El Gobierno de la educación superior en Europa : políticas, estructuras, financiación y personal académico / Eurydice. Madrid : Secretaría General Técnica, Subdirección General de Documentación y Publicaciones. 
Ministerio de Educación (2010) La educación superior en Europa 2009: progresos en el Proceso de Bolonia / Eurydice. Madrid : Ministerio de Educación, Secretaría General Técnica, D.L.

Rué, J. (2007). Enseñar en la universidad: el EEES como reto para la educación superior. Madrid: Narcea.

\section{Nota biogràfica}

$M^{a}$ Elena Añel Cabanelas, es Diplomada en Magisterio y Licenciada con grado en Psicopedagogía. Funcionaria del Cuerpo de Maestros de Pedagogía Terapéutica de la Consellería de Educación y Ordenación Universitaria de la Xunta de Galicia. Estudiante de tercer ciclo de la Universidad de Vigo en la fase de elaboración de Tesis Doctoral. Colaboradora en distintas investigaciones relacionadas con las Tecnologías de la Información y la Comunicación en educación. En pasados cursos escolares profesora invitada del Departamento de Didáctica, Organización Escolar y Métodos de Investigación en la materia de "Nuevas Tecnologías Aplicadas a la Educación" de la titulación de Magisterio en las especialidades de Educación Especial y Educación Física en los Campus de Ourense y Pontevedra respectivamente.

Correspondencia con la autora: C/ San Rosendo, $n^{\circ} 1,2^{\circ}$ B; 32001- Ourense; Tel.: 620.396.622; Correo electrónico: elena_ou@uvigo.es 